\title{
A dedicated superbend x-ray microdiffraction beamline for materials-, geo- and environmental sciences at the Advanced Light Source
}

\author{
Martin Kunz ${ }^{1)}$, Nobumichi Tamura ${ }^{1)}$, Kai Chen ${ }^{1,2)}$, Alastair A. MacDowel1 ${ }^{1)}$, Richard S. Celestre ${ }^{1)}$, \\ Matthew M. Church"), Sirine Fakra"), Edward E. Domning ${ }^{1)}$, James M. Glossinger ${ }^{1)}$, Jonathan L. \\ Kirschman $^{1)}$, Gregory Y. Morrison ${ }^{1)}$, Dave W. Plate ${ }^{1)}$, Brian V. Smith ${ }^{1)}$, Tony Warwick ${ }^{1)}$, Valeriy V. \\ Yashchuk $^{1)}$, Howard A. Padmore ${ }^{1)}$ and Ersan Ustundag ${ }^{3)}$. \\ ${ }^{1)}$ Lawrence Berkeley National Laboratory, 1 Cyclotron Road, Berkeley, CA 94720, USA \\ 2) Department of Materials Science and Engineering, UCLA, Los Angeles, CA 90095 \\ ${ }^{3)}$ Iowa State University, Materials Science and Engineering, 3273 Gilman Hall, Hoover Ames, IA \\ 50011 USA \\ *Email:mkunz@lbl.gov
}

\section{Synopsis}

A new facility for microdiffraction strain measurements and microfluorescence mapping has been developed at the Advanced Light Source. Details of the mechanics and performance of the beamline and endstation will be given.

\section{Abstract}

A new facility for microdiffraction strain measurements and microfluorescence mapping has been built on beamline 12.3.2 at the Advanced Light Source (ALS) of the Lawrence Berkeley National Laboratory (LBNL). This beamline benefits from the hard $\mathrm{x}$-radiation generated by a 6 Tesla superconducting bending magnet (superbend). This provides a hard x-ray spectrum from $5 \mathrm{keV}$ to $22 \mathrm{keV}$ and a flux within a $1 \mu \mathrm{m}$ spot of $\sim 5$. $10^{9}$ photons per seconds $(0.1 \%$ bandwidth at $8 \mathrm{keV})$. The radiation is relayed from the superbend source to a focus in the experimental hutch by a toroidal mirror. The focus spot is tailored by two pairs of adjustable slits, which serve as secondary source point. Inside the lead hutch, a pair of Kirkpatrick-Baez (KB) mirrors placed in a 
vacuum tank re-focuses the secondary slit source onto the sample position. A new KB-bending mechanism with active temperature stabilization allows for more reproducible and stable mirror bending and thus mirrorfocusing. Focus spots around $1 \mu \mathrm{m}$ are routinely achieved and allow a variety of experiments, which have in common the need of spatial resolution. The effective spatial resolution $(\sim 0.2 \mu \mathrm{m})$ is limited by a convolution of beam size, scan-stage resolution and stage stability. A 4-bounce monochromator consisting of 2 channel-cut Si(111) crystals placed between the secondary source and KB-mirrors allows for easy changes between whitebeam and monochromatic experiments while maintaining a fixed beam position. High resolution stage scans are performed while recording a fluorescence emission signal or an x-ray diffraction signal coming from either a monochromatic or a white focused beam. The former allows for elemental mapping, whereas the latter is used to produce 2-dimensional maps of crystal-phases,-orientation, -texture and -strain/stress. Typically achieved strain resolution is in the order of $5 \cdot 10^{-5}$ strain units. Accurate sample positioning in the x-ray focus spot is achieved with a commercial laser-triangulation unit. A Si-drift detector serves as a high-energy-resolution $(\sim 150 \mathrm{eV}$ FWHM) fluorescence detector. Fluorescence scans can be collected in continuous scan mode with up to 300 pixels per second scan-speed. A CCD area detector is utilized as diffraction detector. Diffraction can be performed in reflecting or transmitting geometry. Diffraction data are processed using XMAS, an in-house written software package for Laue and monochromatic microdiffraction analysis.

\section{Keywords: $x$-ray beamline, $x$-ray microdiffraction, $x$-ray fluorescence, superbend.}

\section{Introduction}

The Advanced Light Source (ALS) is a relatively low-energy $(1.9 \mathrm{GeV}), 3^{\text {rd }}$ generation synchrotron optimized for the production of VUV and soft x-ray light from undulators. However, local demands chiefly from the protein crystallography community required the development of hard x-ray sources at the facility. As a result, three 6 Tesla superconducting bending magnets replaced three 1.2 Tesla regular bending magnets ${ }^{1,2}$. This resulted in a shift in the critical energy for these three sources from $3 \mathrm{keV}$ to $12 \mathrm{keV}$ allowing for the development of various hard $\mathrm{x}$-ray programs. The success of the initial x-ray microdiffraction program on the limited energy range provided by the warm bending magnet of beamline 7.3.3 motivated the move of this program to one of the three superbend sources. The recent emergence of x-ray microdiffraction techniques is linked to the increased availability of high brilliance synchrotron sources as well as progress in x-ray focusing optics that nowadays allows obtaining hard x-ray beams with full-width at half-maximum (FWHM) size in the 
order of a few tens of nanometers ${ }^{3-6}$. $\mathrm{x}$-ray microdiffraction is performed both in polychromatic and monochromatic mode.

Synchrotron polychromatic scanning x-ray microdiffraction is a technique that proves to be useful for studying mechanical properties of materials at length scales of the so-called mesoscale (100 $\mathrm{nm}-10$ microns), i.e. in the range of the size of the constitutive grains and defect interactions. The technique is based on raster-scanning the sample with a highly focused (sub-micron) white synchrotron x-ray beam. At each step a 2-dimensional diffraction pattern (Laue pattern) is recorded. The proper analyses of the resulting arrays of Laue patterns allows for reconstruction of maps of grain orientation, strain/stress and dislocation density. The spatial resolution of such maps is limited by the X-ray beam spot size as well as the step size used during the data collection.

This technique has been applied to study the interactions between grains during a tensile loading of a freestanding Al-film ${ }^{7}$. It was also used to show that grains deform plastically at a very early stage of electromigration in $\mathrm{Al}$ and $\mathrm{Cu}$ interconnects ${ }^{8,9}$. A third example for the successful application of microdiffraction is the demonstration of grain rotation effects in Sn-lines subject to electromigration, which in turn explains their sudden resistivity drop ${ }^{10}$. Polychromatic $\mathrm{x}$-ray microdiffraction is complementary to monochromatic $\mathrm{x}$-ray microdiffraction. Monochromatic scanning x-ray microdiffraction is mainly used for phase identification through powder diffraction patterns in highly heterogeneous samples with sub-micron grain size. Most of these applications are found in geological, environmental or archaeological studies ${ }^{11-13}$.

X-ray microdiffraction offers important advantages over charged particles techniques such as electron microscopy and focused ion beam microscopy in that the radiation used is more penetrating and probes bulk and buried materials at depths of $10 \mu \mathrm{m}$ to $1 \mathrm{~mm}$, depending on chemical composition of the sample investigated and the available x-ray energy range. Also, the strain and grain orientation measurements with this technique provide for better resolution than those obtained by charged particle techniques.

There are a few drawbacks to this technique. The first one is its low availability. This is addressed by the ongoing development of new dedicated microdiffraction beamlines at synchrotron sources around the world. Beamlines offering micron to submicron beam sizes are now available at several synchrotron facilities, including the ALS (Berkeley, USA), APS (Chicago, USA), ESRF (Grenoble, France), PLS (Pohang, South-Korea) and SLS (Villigen, Switzerland) and some are in construction as at the CLS (Saskatoon, Canada) and in the planning phase as at SOLEIL (Paris, France) and the Australian Light Source (Melbourne, Australia).

A second drawback is a possible limitation in flux, which varies between facilities. Undulators are perfectly suited for monochromatic experiments but are not ideal for white beam experiments due to their highly structured energy spectrum. While white beam experiments are feasible to some extend with undulators by 
tapering the gap or aligning them off-axis, they are most easily performed on wiggler or bending magnet beamlines. The advent of superconducting bending magnets, as will be described in this paper, represents thus important progress for this technique.

A third drawback in the case of white beam Laue experiments is the complexity of the analysis required to extract meaningful information, such as crystal orientation, strain and stress tensors or defect densities. Deformed samples often exhibit diffraction peaks with shapes far from ideal Lorentzian profiles. Instead, they are streaked in various directions, which make them substantially more difficult to analyze than e.g. Kikuchi patterns from electron back-scattering diffraction (EBSD). Also, several grains can simultaneously satisfy the

Bragg condition in thicker samples with high penetration depth or in the vicinity of grain boundaries. These problems can be overcome if depth resolved Laue patterns are available.

Another issue being addressed is spatial resolution, since it is much harder to focus hard x-rays than it is for charged particles. However, continued progress in the design and fabrication of x-ray optics such as KirkpatrickBaez $(\mathrm{KB})$ mirrors is improving the resolution into the $\mathrm{nm}$ range. In fact, currently achievable $\mathrm{x}$-ray focus spots are in the range that resolution is not so much limited by the dimension of the focus spot, but much more by residual vibrations in the $100 \mathrm{~nm}$ range of the sample stage assemblies.

In this work we describe a new facility for x-ray microdiffraction and x-ray microfluorescence, which has been built on beamline 12.3.2 at the ALS (LBNL). Section 2 describes the characteristics of the source. The details of the beamline design and the control system are presented in sections 3 and 4, respectively. Beamline performance and future plans are discussed in chapters 5 and 6.

\section{Source}

The three 6 Tesla superconducting bending magnets have been operating continuously since their installation in the ring lattice in 2002. They have been transparent to the users and can be viewed as a technical success that allows the ALS to have inexpensive hard x-ray bending magnet sources and the development of the associated hard x-ray programs. The design allows for 4 beamlines per magnet, with inboard and outboard pairs of tangent points at field strengths of 4.37 and 5.29 Tesla, respectively. These fields increased the critical energy from $3 \mathrm{keV}$ for a 1.27 Tesla normal conducting magnet to 11.5 and $12.7 \mathrm{keV}$, respectively. The micro-diffraction beamline described in this work has a source critical energy of $11.5 \mathrm{keV}$. The beam size in the ALS is small, due to the small emittance of the electron beam $(6.3 \mathrm{~nm}-\mathrm{rad}[\mathrm{h}] ; 0.13 \mathrm{~nm}-\mathrm{rad}[\mathrm{v}])$ and the small beta functions $(0.95 \mathrm{~m}$ $[\mathrm{h}] ; 1.5 \mathrm{~m}[\mathrm{v}]$ ). Together with the small dispersion at the dipole position (dispersion $=0.57 \mathrm{~m}$; slope of dispersion 
$=-0.04)$, this yields electron beam sizes of $230 \mu \mathrm{m}[\mathrm{h}]$ by $35 \mu \mathrm{m}[\mathrm{v}](\mathrm{FWHM})$, which is the source size for this beamline.

\section{Beamline design}

The design of the beamline had to satisfy the following requirements: 1) Easy switch from white beam conditions to a monochromatic set-up while accurately maintaining the beam position. 2) The monochromator needs to be able to scan in energy with a constant beam position without dropping off the rocking curve. 3) The sample needs to be scanned through the beam in two directions with an accuracy of at least $100 \mathrm{~nm}$.

The layout for this beamline (beamline 12.3.2 in ALS nomenclature) is depicted schematically in Figure 1: It consists of the source, a horizontally deflecting toroidal M1-mirror (grazing angle $3.5 \mathrm{mrad}$ ), a pair of roll slits defining the size of the virtual secondary source, a 4-bounce two-channel-cut $\mathrm{Si}(111)$ monochromator, a second pair of slits (from JJ-X-ray, Denmark) serving as aperture, a set of Kirkpatrick-Baez (KB) mirrors, the sample stage and two detectors. The acceptance of the M1-mirror is limited to $0.2 \mathrm{mrad}$ by a water-cooled aperture. The distances from the source for this beamline are $13 \mathrm{~m}, 22.4 \mathrm{~m}$ and $24.8 \mathrm{~m}$ for M1, secondary source slits and sample, respectively. Limitations by the shield wall and the short distance of the beamline prevent the placement of the M1-mirror at the usual unity magnification position, where aberrations are at a minimum. Instead, its actual placement causes a demagnification of the source by a factor of 0.723 at the secondary source position. The sagittal acceptance of M1 is small, such that aberrations due to the M1 demagnification are small thus reducing the brightness by only $10 \%$ from the $1: 1$ condition. The horizontal and vertical focusing KB mirrors are positioned at $0.27 \mathrm{~m}$ and $0.135 \mathrm{~m}$ upstream of the sample, respectively. This results in demagnification factors of $7.89(\mathrm{~h})$ and $16.78(\mathrm{v})$, respectively.

All elements downstream from and including the secondary source are enclosed in an interlocked lead hutch (2 $\mathrm{mm}$ lead between two $1.9 \mathrm{~mm}$ steel plates). Monochromator, aperture slits and KB mirrors are further contained in an aluminium box, which is kept at a vacuum of about $10^{-5}$ mbar. The vacuum is maintained by combining a dry roughing pump with a turbo pump. To avoid temperature related shifts of the sample with respect to the beam due to the thermal expansion of the various beamline components, the temperature inside the hutch is maintained constant within $0.2^{\circ} \mathrm{C}$.

\subsection{Optics}




\subsubsection{Primary focusing mirror}

The silicon M1 mirror has a length of $700 \mathrm{~mm}$, a width of $75 \mathrm{~mm}$ and a thickness of $75 \mathrm{~mm}$ and is coated with $25 \mathrm{~nm}$ of Pt under $8 \mathrm{~nm}$ of Rh. The additional Rh layer suppresses the Pt absorption edges between 10 and 13 $\mathrm{keV}$, but maintains high reflectivity at higher energies as these $\mathrm{x}$-rays reflect from the Pt layer. The mirror can be adjusted in pitch, yaw and two bends (upstream and downstream). Thermal load calculations indicate that with such a configuration no cooling of the mirror is required as the total power absorbed by the mirror will be only $1.77 \mathrm{~W}$. In practice we find the M1 to be stable with a beam position stability $<10 \%$ of the source size. The size of the primary focus point (secondary virtual source) is controlled by two pairs of water-cooled tungsten rods orientated in the horizontal and vertical, respectively. Each pair of rods is separated by a 2 mm gap through which the beam passes. The rods are mounted on a rotary stage, which, when turned closes down this $2 \mathrm{~mm}$ gap to micron dimensions required to define the secondary source. This is the first element within the lead hutch. The size of the secondary source size has been calculated to be $175 \mu \mathrm{m}$ (h) by $30 \mu \mathrm{m}(\mathrm{v})$. Measurements show slightly larger beam spot of $190 \mu \mathrm{m}(\mathrm{h})$ by $36 \mu \mathrm{m}(\mathrm{v})$ at the roll slits. In order to achieve a final focus spot of $1 \mathrm{x}$ $1 \mu \mathrm{m}$ with the present geometry, the secondary source has to be slitted down to $8 \mu \mathrm{m}$ (h) to $16 \mu \mathrm{m}(\mathrm{v})$. This causes a loss in flux by a factor of $\sim 50$ relative to the flux accepted by the M1 mirror.

\subsubsection{Kirkpatrick-Baez mirrors}

A pair of bendable mirrors in Kirkpatrick-Baez $(\mathrm{KB})$ configuration provides the micro-focus spot. The mirror substrates are made of a super polished plane single crystal of silicon of $102 \mathrm{~mm}$ (length) x 8-13 mm (width) x 4 mm (thickness). The substrates are coated with $25 \mathrm{~nm}$ Pt under $8 \mathrm{~nm} \mathrm{Rh}$. The substrate width has been carefully profiled wider in the middle to correct for higher order errors between the desired elliptical shape and that achievable by simple beam bending alone ${ }^{14}$. Each mirror has 4 degrees of freedom: one linear translation to position the mirror relative to the beam, one pitch to adjust the incident angle and thus focal length and two bends, one at each end, to set the elliptical curvature of the mirrors. Each degree of freedom can be remotely driven with picomotors. Absolute position feedback is provided by a linear variable differential transformer (LVDT) on each of the picomotors. An additional picomotor applied to the vertical KB mirror is used to correct the orthogonality between the two mirrors. In order to stabilize the mirror temperature, the temperature of the mirror bases is controlled with a Peltier module. The heat flows from the ends of the mirror substrate through the flexural assembly to the Peltier thus stabilizing the substrate temperature and minimizing undesirable strains that heat straps might induce. Temperature of the mirrors inside the vacuum tank is maintained constant within $<0.1$ ${ }^{\circ} \mathrm{C}$. The mirrors are mounted on partially constrained flexural supports allowing one end to float longitudinally to 
adjust for mirror length changes and the other end to twist axially to remove twist from the longitudinal axis. Picomotors apply moments to the ends of the mirror via a weak leaf spring pulled by a thin flexible wire to demagnify actuator motion and minimize the effects of thermal expansion and off axis motions of the linkage assembly. The mirrors were bent to desired shape to an accuracy of $\pm 0.5 \mu$ rad with the aid of a long-traceprofiler in the optical metrology lab ${ }^{15}$ prior to installation at the beamline.

In addition, a simple system for quick online-optimization of bend and pitch based on the Hartmann Test $^{16}$ has been developed. A Hartmann test applied to the reflective focusing optics of the KB mirror works such that different parts of the KB mirror are successively illuminated and the changes of the spot position at the focal point are recorded as a function of the illuminated part. We expect an error in the mirror pitch to be expressed by a linear dependence of the spot position on the illuminated path. An erroneous shape of the mirror stemming from non-optimal bend setting, on the other hand, should lead to a curvature in the plot. We use the aforementioned high precision aperture slits (JJ X-Ray) closed to a size of 5 microns and placed $\sim 10 \mathrm{~cm}$ upstream the $\mathrm{KB}$ assembly to act as a pinhole that can be translated vertically and horizontally within the acceptance area of the $\mathrm{KB}$ focusing system. The position of the doubly reflected beam at the focal point is recorded using a device consisting of a scintillator placed at the focal point and a high magnification CCD camera. The scintillator that converts x-ray into visible light is a $1 \mathrm{~mm}$ thick piece of single crystal $\mathrm{CdWO}_{4}$. The CCD camera capturing the beam image on the scintillator is equipped with a zoom lens (Edmund Optics VZM450). Aluminium foils need to be placed upstream of the scintillator to attenuate the x-ray beam in order to prevent saturation of the signal on the scintillator. We found that it is necessary to precisely focus the lens onto the scintillator to minimize errors on the beam position. The camera is therefore mounted on a remotely controlled stage. With this technique, mirror figure errors of $0.2 \mu \mathrm{rad}$ can be measured and if they stem from wrong bend settings can be corrected for. A software code developed in-house ALS ${ }^{17}$ is used to optimize the bends with only a few iterations. Figure 2 shows the result of an online Hartmann scan after pitch and bend optimization for different bend configurations. The horizontal line indicates that the focussed spot does not move as the mirror surface is probed by means of tracking the beam across the mirror surface. Deviation from a horizontal line is a slope error plot of the mirror surface.

\subsubsection{Monochromator}

In order to be able to easily switch between white and monochromatic mode, a four-bounce $\operatorname{Si}(111)$ monochromator was installed upstream of the aperture slits and KB mirror pair. Figure 3 gives basic dimensions 
of the monochromator. The monochromator consists of two channel cut $\mathrm{Si}(111)$ crystals mounted on separate rotation stages equipped with Renishaw optical linear encoders. The stages are rotated through a sine-bar mechanism with two linear actuators pushing against a steel bar attached tangentially to the rotary stages. The crystals are mounted on the rotary stages, such that the rotation axes lie in the diffracting plane of the $2^{\text {nd }}$ and $3^{\text {rd }}$ crystal respectively (Figure 3). In this geometry, the coordinated, but opposite rotation of the monochromator crystals allows for energy change while keeping the beam at a constant position, which is identical to the white beam position. The first crystal is cooled through a Peltier element, which in turn is water-cooled. Measurements show a slight energy dependence of the angular settings for the two crystals, which is different for both crystals. This results in a linear shift of the energy calibration with energy, as well as a linear shift of the angular off-set of crystal-2 relative to crystal-1 (rocking curve off-set). Both effects are related to the non-planarity of the diffracting surface. This is caused by non-homogenous etching when the (recycled) crystals were prepared for installation in beamline 12.3.2. Since both effects are linear with angle and seem to be constant over time, they are corrected for by calibrating the relevant correction factors and implementing them into the control software.

The non-planarity of the monochromator surfaces also leads to a reproducible small vertical off-set of the monochromatic beam, relative to the white beam, of $2 \mu \mathrm{m}$. This error is compensated for by the precision sample stage described next.

\subsection{Sample Stage:}

The sample is positioned on a high-precision stage consisting of 8 different motorized stages as indicated in Figure 4 The lower xyz stage allows for aligning the rotation axes $(\chi$ and $\phi)$ onto the $\mathrm{x}$-ray focal point. The upper xyz stages allow to place the sample onto the center of rotation and to scan the sample through the x-ray beam. Since the beamline is designed for high spatial resolution with an X-ray focus spot below 1 micron, the scanning stages need to be more precise than this. We use two linear micro-stepping stages (Micos PLS-85) with $50 \mathrm{~nm}$ resolution Heidenhain linear encoders to fulfill these requirements. The $\chi$ partial arc allows for easy change between reflection $\left(\chi=45^{\circ}, \mathrm{CCD}\right.$ center $\left.=90^{\circ}\right)$ and transmission mode $\left(\chi=0^{\circ}, 0^{\circ}<\mathrm{CCD}\right.$ center $<90^{\circ}$ ). The sample is viewed through an Infinity K2 long working distance microscope with variable magnification and a video camera. The maximum magnification of the viewing system is $1.6 \mu \mathrm{m} / \mathrm{CCD}$-pixel.

The sample is accurately positioned on the previously calibrated (knife edge scans) x-ray focus point by means of a commercial laser triangulation unit (Keyence LK-G152). The system works such that the diffuse image of the laser spot $(0.95 \mathrm{~mW} 650 \mathrm{~nm}$ class II diode laser) is imaged onto a linear CCD unit. The relative positions of the CCD-unit and the laser-emitting diode are fixed and known. The position of the laser image on 
the CCD-unit thus measures the angle between the incident laser beam and the diffusely reflected image. This in turn determines the distance from the unit to the image forming surface. The system allows for positioning with an accuracy of below $\sim 1 \mu \mathrm{m}$, i.e. about 2 orders of magnitude better than required by the convergence of the $\mathrm{x}$ ray beam (2.6 mrad vertical, $1.3 \mathrm{mrad}$ horizontal).

The absolute distance between sample and diffraction-detector is additionally calibrated using a Sidiffraction standard single crystal. The precisely known cell-parameters allow for calibration of sample to detector distance together with other instrument parameters such as detector tilt and the center of the CCDdetector. Since the calibration is done using a quasi-perfect crystal of $\mathrm{Si}$ (thickness $\sim 500 \mu \mathrm{m}$ ), the penetration of the X-rays into the crystal and its effect on the calibration are negligible. Tests on crystals of different thicknesses and with or without penetration correction did not show any significant difference in the calibrated parameters.

Vibration control of the experimental stage is critical. This is always a problem for experimental stations at synchrotron sources as the source is fixed and the usual vibration isolating pneumatic supports lack adequate positional stability to be used for vibration isolation. As a result this experimental station is mounted hard from the floor. The optical layout within the hutch, sample stage and CCD goniometer are mounted on a regular optical bench which in turn is mounted hard off the floor. The usual design considerations are employed whereby items are designed to be stiff and of low mass to drive the resonant frequencies to high values where amplitude excursions are less. Considering this, the vibrational modes of the present stage configuration were determined using a commercial accelerometer (SignalCalc Mobilyzer) as well as a laser Doppler shift vibrometer (Polytec Inc.; OFV-552 Fiber-Optic Interferometer). Both, accelerometer and vibrometer can only detect vibrations between the different components of the beamline but not vibrations of the beam itself. In addition, the intensity variation of the $\mathrm{x}$-ray beam with a knife-edge (positioned on the sample stage) shadowing $50 \%$ of the beam profile was frequency analyzed. This gives us the frequency distribution of the stage-vibration relative to the x-ray beam. Vibrations measured with this arrangement show strong horizontal variation with main frequencies at $15-30 \mathrm{~Hz}, 40 \mathrm{~Hz}, 45 \mathrm{~Hz}$ and $90 \mathrm{~Hz}$ (Figure 5). These measurements are the most meaningful as they integrate all the vibrations as seen by the sample with respect to the beam. Of these modes, the 40 and 45 $\mathrm{Hz}$ mode seem to be connected to vibrations on the sample stage as they are also present in the accelerometer measurements performed on the sample stage, but absent or weak on measurements made on the KB box or the roll slits stand. The $25 \mathrm{~Hz}$ mode is present in all measurements made on the stages, KB box and roll slits, indicating that it is most likely associated with vibrations of the ALS experimental floor. The $90 \mathrm{~Hz}$ contributions seem to be linked to movements of the roll slits, which translates into beam motion at sample stage 
and is only present in measurements performed on the roll slits and the knife-edge. Of all the frequencies, the largest amplitudes come from the 40 and $45 \mathrm{~Hz}$ associated with the sample stage in the horizontal direction, meaning that the stage is swinging in the $\mathrm{x}$-direction. Mechanical coupling of sample stage to the $\mathrm{KB}$ box considerably reduces the magnitude of the vibration, but the FWHM of the measured spot size remained unchanged.

The amplitude of the vibration was measured using a fast camera. These results show a FWHM of the vibrational amplitude around $0.5 \mu \mathrm{m}$. The spot size limit is presently around $1 \mu \mathrm{m}$ and thus not limited by the vibrations of the system.

\subsection{Detectors:}

The beamline is equipped with two detectors, namely a MAR (now Rayonix) 133 x-rayCCD and a Sidrift detector. The MAR 133 is used to record the polychromatic and monochromatic diffraction patterns. It has a $133 \mathrm{~mm}$ diameter active area, which is tapered down to a $2 \mathrm{k} \times 2 \mathrm{k}$ chip with a single fiber-optic system. By default, the CCD is used in a $2 \times 2$ re-binned $1 \mathrm{k} \times 1 \mathrm{k}$ mode as a best compromise between readout time and resolution. The CCD detector is mounted on a Huber 440 stage with its rotation axis mounted horizontally. This allows placing the detector at various vertical 2-theta angles between $0^{\circ}$ and $90^{\circ}$. Careful commissioning measurements of refined sample-to-detector distances as a function of 2-theta angle of the CCD detector found the center of the 2-theta arm to be about $3.5 \mathrm{~mm}$ upstream and $1.3 \mathrm{~mm}$ below the calibrated x-ray focus spot. This results in a change of the sample to detector distance. Since this offset is constant it can be calibrated and variations of sample to detector distance with changing detector angle can be corrected for without the need to record a separate calibration pattern for each individual detector setting.

The Si-drift detector (Vortex-EM by SII Nanotechnology Inc.) is used for X-ray fluorescence elemental mapping, which in turn can also be used to precisely locate the focused x-ray on the sample, provided a marker with appropriate dimension and fluorescence line is present on the sample surface. The detector has a $50 \mathrm{~mm}^{2}$ single element active area which is capable to handle count rates up to $600 \mathrm{kcps}$ at a resolution of $\sim 150 \mathrm{eV}$ at 6 $\mathrm{keV}$. It is mounted on a translation stage, which allows for remote distance adjustment and thus additional increase of the dynamic range.

\section{Control System and Software.}

Beamline components (including the fluorescence detector) and MAR133 CCD are controlled from different computers and thus control systems. The beamline control is based on LabView running on a Windows 
platform. This system is used on most ALS beamlines, which allows quickly transferring software developments on a given beamline to other stations. At the same time, beamline specific applications can be added to the base software in a modular way. For the microdiffraction beamline a fast fluorescence mapping software as well as a 2-dimensional video scanning routine has been added. The former allows for very rapid collection of fluorescence maps with several $10^{5}$ map-points. The latter is essential for 2-dimensional scanning of video captured images of the x-ray beam for e.g. mirror bend optimization and Hartmann tests.

The x-Ray CCD is controlled with MAR proprietary software running on a Linux station. For diffraction scans, an IDL and Fortran based, in-house written control software (XMAS) interfacing the CCD control with the Labview motor control has been added ${ }^{18}$. Besides acting as interface in the diffraction scan process, XMAS is also the primary data reduction and analysis software used on the beamline. It allows for system calibration (sample-to-detector distance, detector tilt angles, detector center), background correction, indexing (provided relative cell parameters are known), as well as strain and stress tensor refinements. Depending on data quality and size and symmetry of unit cells in question, up to 100 individual grains can be differentiated and indexed in a given pattern. XMAS can be used in an interactive file-by-file mode or in an automatic mode where several thousands of diffraction files scan can be sequentially analyzed automatically.

While XMAS is designed for Laue pattern analysis, it can also be used for monochromatic powder diffraction data, where it serves to compare recorded powder diffraction patterns with known phases. A cluster-based version of XMAS has also been developed running on a 24 nodes dual processors Linux cluster.

\section{Beamline Performance}

Beamline 12.3.2 is fully operational since summer 2007 and accepts user proposals since August 2007. While a minimal spot-size of $0.6 \mu \mathrm{m}(\mathrm{h}) \times 0.5 \mu \mathrm{m}(\mathrm{v})$ (FWHM) was achieved in the initial commissioning period, user experiments are presently run with $\mathrm{a} \sim 1 \mu \mathrm{m} \times 1 \mu \mathrm{m}$ beam. This spot size can be reproduced on a routine basis and seems not to degrade over the time-period observed. The initial decline of the mirror performance is probably due to the degradation of the mirror coatings due to the fact that for practical reasons, many of the test runs during the initial commissioning period were performed with nitrogen flow instead of vacuum inside the enclosure. Spot sizes are measured by scanning a tungsten-knife-edge through the beam at the focal point and measuring the change in recorded x-ray intensities with a PIN diode. Figure 6 show the results of spot size measurement obtained with a $150 \mu \mathrm{m}$ diameter tungsten wires used as a knife-edge. Beam intensities were collected via a calibrated Pin diode. 
The calculated and measured fluxes arriving at the sample are shown in Figure 7. The electron source used has an energy of $1.9 \mathrm{GeV}$ within a field of 4.37 Tesla at the tangent point. The absolute flux was measured by using an ion chamber (IC). The IC has a total length of $65 \mathrm{~mm}$, an active length of $50 \mathrm{~mm}$ and is filled with 1 bar of Ar gas. The current measured in the ion chamber is converted into number of x-ray photons, using a conversion factor of $26 \mathrm{eV}$ of energy per electron - ion pair $^{19}$. The result of the flux measurements is compared against the expected values in Figure 7. The calculated total flux from the Si(111) crystals is about an order of magnitude higher than the one measured. The reason for this discrepancy is still under investigation. We suspect the aforementioned etching induced irregularities on the monochromator planes to induce walk-off effects on the individual planes thus affecting the monochromator efficiency. For the time being, the two individual channelcut crystals cannot be tuned onto each other online with respect to their position. Subtle alignment errors can have significant effects in combined reflectivity, especially at high energies, i.e. low angles.

With the achieved spot size in combination with the high-resolution x-y scanning stage and the energy spectrum of the superconducting bending magnet, strain resolutions of $5 \cdot 10^{-5}$ strain units at a spatial resolution of $0.2 \mu \mathrm{m}$ are routinely achieved. Such strain resolution is achieved by averaging out the errors on peak positions over the whole Laue diffraction patterns. The beam convergence on the sample is $1.3(\mathrm{~h})$ and $2.6(\mathrm{v}) \mathrm{mrad}$, respectively, so that each diffraction peak spreads over several pixels on the detector, allowing to obtain subpixel resolution on peak position by $2 \mathrm{D}$ peak fitting and/or centroiding. The spatial distortion is corrected within the MAR acquisition software and shown to be insignificant after correction (as tested on a piece of perfect silicon crystal). Note that scanning a $1 \mu \mathrm{m}$ beam in sub-micrometer steps allows for higher spatial resolution of the strain maps than given by the beam size. When switching from white light to monochromatic beam, an intensity loss of $\sim 4$ orders of magnitude is observed. For experiments involving weakly diffracting samples (environmental science), this loss of intensity is detrimental but can be compensated by opening the virtual source and thus using a larger spot size $[\sim 5 \mu \mathrm{m}(\mathrm{h}) \times 2 \mu \mathrm{m}(\mathrm{v})$ up to $\sim 20 \mathrm{um}(\mathrm{h}) \times 2$ um (v)]. In those cases monochromatic scans are thus spatially less resolved than white-beam scans. The upcoming upgrade of the ALS storage ring to top-off mode and its operation at a higher electron current of $500 \mathrm{~mA}$ helps alleviate some of these drawbacks of the monochromatic beam operational mode. Scans in white beam mode are presently limited by the readout time of the X-ray CCD detector ( 5 seconds). The overhead time contributes significantly more to the total scan-time in white-beam mode, where typical exposure times are presently in the order of tenths of seconds, whereas in monochromatic mode, exposure times in the order of tens of seconds to minutes dominate 
the total experimental time. We expect exposure times to be reduced up to a factor of 0.5 when operating in topoff mode.

The beamline is presently mainly used for material science studies related to the micromechanics and reliability issues of technologically interesting manufactured samples. One such example is the study of electromigration in $\mathrm{Pb}$-free $\mathrm{Sn}$-based solder joints used in flip-chip technologies. Current crowding ${ }^{20}$ leads to localized high current densities at the corner where the electrons enter into or exit from the solder bumps, so that Sn whiskers and pancake-type voids are formed at the anode and cathode end, respectively ${ }^{21}$. The Sn-based solder joints have body-centred tetragonal crystal structure. It's thermal and electric conductivity, following Neumann's principle, are therefore anisotropic properties: the electric conductivity in the crystallographic cdirection is significantly lower than in $\mathrm{a} / \mathrm{b}$-direction ${ }^{10}$. Grain orientation has been monitored during an accelerated in-situ electromigration test using white-beam x-ray microdiffraction. The results showed that the grains in the current crowding region were reoriented after exposure to high current densities (Figure 8). The sense of rotation is such that it realigns the crystallographic a-direction along the electron flow direction which lowers the effective resistance of the grains. Figure 9 shows the evolution of the effective conductivity as a function of time. Here the conductivity has been derived from the orientation of the Sn-grains ${ }^{22}$.

Another example using white beam microdiffraction is the study of the orientation of aragonite grains at the interface between mother-of-pearl and prismatic calcite layers in an abalone $\operatorname{shell}^{23}$. Mother of pearl (Nacre) can be viewed as a natural composite material built of stacks of aragonite crystals linked with organic matrix (mostly $\beta$-chitin). It is remarkable in that its strength surpasses that of its primary building material (Aragonite) by a factor of 3000 , due to its accurately self-assembled architecture. The goal of this study was to better quantify the architecture of the nacre composite on the length scale of the individual crystallites. White beam xray microdiffraction measurements on beamline 12.3.2 confirm and add quantitative information to linear dichroism measurements under a PEEM microscope. They show that the orientational ordering of the aragonite crystals increase with increasing distance from the calcite to mother-of-pearl interface with an initial spread of the orthorhombic c-axis of $\sim 30^{\circ}$ to less than $10^{\circ}$ at $35 \mu \mathrm{m}$ distance from the interface (Figure 10). These data are consistent with a theoretical model in which aragonite crystal layers are nucleated sequentially in the presence of confining matrix sheets and grow epitaxially on aragonite crystals in layers below, with the aragonite tablets with its crystallographic c-axes oriented normal to the layers growing faster than misoriented tablets.

Monochromatic beam experiments are typically used for phase identifications and mineralogical mapping. One such example is the identification of secondary nanocrystalline phases during the passivation process of acid mine water ${ }^{24}$. The standard treatment of acid mine water involves an anoxic limestone drainage 
through which the acid water percolates. Dissolution of calcite leads to a buffering of the acidity and thus significant increase of the $\mathrm{pH}$. Re-precipitation of secondary phases over time, however, leads to a passivation of these systems. The goal of this particular study was to quantify the kinetics of this process and to understand the nature of the secondary precipitates, which coat the calcite grains and thus passivate the buffer zone. Monochromatic x-ray microdiffraction measurements along the calcite grains showed unambiguously that gypsum $\left(\mathrm{CaSO}_{4} \mathrm{H}_{2} \mathrm{O}\right)$ acts as the first coating material on the surfaces of the calcite grains. Fe-bearing phases (mostly goethite $(\mathrm{FeOOH})$ ) fill interstitials between the grains but are not in direct contact with the calcite grains.

\section{Future Developments}

In its present configuration, beamline 12.3.2 produces 2-dimensional strain/stress maps and phase identification on a routine basis. This, however, exploits only a part of the potential provided by a micro-focused white and/or monochromatic synchrotron beam. In particular we envisage the expansion of the 2-dimensional strain mapping into 3-dimensions by including depth resolution. We are adopting a two-pronged approach. On the one hand we intend to implement the wire scan method as developed by Ref. 25. This requires a large amount of data-collection and analysis time. In cases where the requirements for depth resolution are less stringent, an alternative method based on depth triangulation using the variable position of an x-ray reflection on the area detector as a function of the detector-distance is being implemented. While this doesn't give the depth resolution achieved by the wire scan method, it has the advantage that it is much faster and computationally less demanding, thus allowing for larger areas to be covered. Efforts in these directions had been initiated on beamline 7.3.3 $3^{26}$ before the shutdown of its microdiffraction program in 2006 and are being continued on 12.3.2, the goal being to implement these techniques in a fully user-friendly fashion. One of the problems inherent to either method will be the relatively limited high-energy range of 12.3.2 which will pose limits on the penetration depth, especially for high-Z material.

Two-dimensional as well as three-dimensional strain / stress scanning only makes use of the peak positions. The information provided by the peak intensities is largely ignored. Interpreting intensities may be useful in cases where traditional monochromatic diffraction methods (powder or single crystal) are not applicable ${ }^{27,28}$. This could occur in the case of rare and very small crystals which cannot be isolated and mounted or where bulky and immobile ancillary equipment prohibit the 3-dimensional rotations required for a monochromatic single crystal dataset. The application of a white x-ray beam on a static sample compensates for the lack of mobility without losing the three dimensional information about the reciprocal lattice, as it happens in 
the case of monochromatic powder diffraction. The problems to be solved are the determination of the various wavelength dependent correction factors and the absolute indexing of a substance with unknown unit cell. First steps to solve the former challenge were undertaken by using well-known standard crystals (Silicon, Ylid) and comparing its expected intensities (based on their known structure as well as analytical polarization and absorption corrections) with the observed intensities. To address the absolute indexing problem we have started developing procedures and software involving monochromator scans. These first efforts produced encouraging results. First experiments on Zeolite micro-crystals revealed a series of experimental issues, such as improved background reduction and optical imaging, which have to be addressed to enhance Laue intensity collection.

\section{Conclusions}

Beamline 12.3.2 of the Advanced Light Source provides for a versatile micro-focus beamline allowing for strain/stress mapping as well as phase identification with high spatial resolution. The increased energy range up to $22 \mathrm{keV}$ delivered by the superconducting magnet provides strain resolution as small as $5 \cdot 10^{-5}$ strain units. The beamline can be operated in white-light mode as well as in monochromatic mode with an extremely easy switch in less than 2 minutes between the two set-ups. This allows for experiments combining phase identification of nano-crystalline material and strain/stress and orientation mapping of micro-crystalline samples.

\section{Acknowledgements}

The Advanced Light Source is supported by the Director, Office of Science, Office of Basic Energy Sciences, Materials Sciences Division, of the U.S. Department of Energy under Contract No. DE-AC02-05CH11231 at Lawrence Berkeley National Laboratory and University of California, Berkeley, California. The move of the micro-diffraction program from ALS beamline 7.3.3 onto the ALS superbend source 12.3.2 was enabled through the NSF grant \# 0416243.

\section{DISCLAIMER}


This document was prepared as an account of work sponsored by the United States Government. While this document is believed to contain correct information, neither the United States Government nor any agency thereof, nor The Regents of the University of California, nor any of their employees, makes any warranty, express or implied, or assumes any legal responsibility for the accuracy, completeness, or usefulness of any information, apparatus, product, or process disclosed, or represents that its use would not infringe privately owned rights. Reference herein to any specific commercial product, process, or service by its trade name, trademark, manufacturer, or otherwise, does not necessarily constitute or imply its endorsement, recommendation, or favoring by the United States Government or any agency thereof, or The Regents of the University of California. The views and opinions of authors expressed herein do not necessarily state or reflect those of the United States Government or any agency thereof or The Regents of the University of California.

\section{References:}

${ }^{1}$ D. Robin, et al., Proceedings of the 2002 European Particle Accelerator Conf., Paris, France, 215 (2002).

${ }^{2}$ L. Tamura and A. Robinson, Synchrotron Radiation News, 15, 30 (2002).

${ }^{3}$ H. Mimura, H. Yumoto, S. Matsuyama, Y. Sano, K. Yamamura, Y. Mori, M. Yabashi, Y. Nishino, K. Tamasaku, T. Ishikawa, and K. Yamauchi, Appl. Phys. Lett. 90(5), 059103 (2007).

${ }^{4}$ H. C. Kang, J. Maser, G. B. Stephenson, C. Liu, R. Conley, A. T. Macrander, and S. Vogt, Phys. Rev. Lett. 96(12), 127401 (2006).

${ }^{5}$ O. Hignette, P. Cloetens, G. Rostaing, P. Bernard, and C. Morawe, Rev. Sci. Instrum. 76(6), 063709 (2005).

${ }^{6}$ W. J. Liu, G. E. Ice, J. Z. Tischler, A. Khounsary, C. Liu, L. Assoufid, and A. T. Macrander, Rev. Sci. Instrum. 76(11), 113701 (2005). 
${ }^{7}$ R. Spolenak, W. L. Brown, N. Tamura, A. A. MacDowell, R. S. Celestre, H. A. Padmore, B. C. Valek, J. C. Bravman, T. Marieb, H. Fujimoto, B. W. Batterman, and J. R. Patel, Phys. Rev. Lett. 90, 096102 (2003).

${ }^{8}$ B. C. Valek, J. C. Bravman, N. Tamura. A. A. MacDowell, R. S. Celestre, H. A. Padmore, W. L. Brown, B. W. Batterman, and J. R. Patel, Appl. Phys. Lett. 81, 4168 (2002).

${ }^{9}$ A.S. Budiman, W.D. Nix, N. Tamura, B.C. Valek, K. Gadre, J. Maiz, R. Spolenak, and J.R. Patel, Appl. Phys. Lett. 88, 233515 (2006).

${ }^{10}$ A. T. Wu, K. N. Tu, J. R. Lloyd, B. C. Valek, K. C. R. Kao, Appl. Phys. Lett. 85, 2490 (2004).

${ }^{11}$ A. Manceau, N. Tamura, R. S. Celestre, A. A. MacDowell, N. Geoffroy, G. Sposito, and H. A. Padmore, Environm. Science and Technol. 37, 75 (2003).

${ }^{12}$ A. Manceau, M. A. Marcus, and N. Tamura, in Reviews in Mineralogy and Geochemistry, Vol. 49, edited by P. A. Fenter, M. L. Rivers, N. C. Sturchio, and S. R. Sutton (Mineralogical Society of America, Washington, DC, 2002) pp. 341-428.

${ }^{13}$ P. Sciau, P. Goudeau, N. Tamura, and E. Dooryhee, Appl. Phys. A-Materials Science and Processing 83, 219 (2006).

${ }^{14}$ M. Howells, S. C. Irick, A. A. MacDowell, H. A. Padmore, T. Renner, S. Y. Rah, and R. Sandler, Optical Engineering 39, 2748 (2000).

15 J. L. Kirschman, E. E. Domning, W. R. McKinney, G. Y. Morrison, B. V. Smith, and V. V. Yashchuk, Photonics 2008: Optical Engineering and Applications Symposium. Conference 7077-10: Advances in Xray/EUV Optics and Components III (2008)

${ }^{16}$ J. Hartmann J, Z. Instrumentenkunde, 20, 47 (1900). 
${ }^{17}$ W. R. McKinney, S. C. Irick, K. L. Kirschman, A. A. MacDowell, T. Warwick, and V. V. Yashchuk, Proc SPIE 6704, 67040G (2007).

${ }^{18}$ N. Tamura et al., J. Synchrotron Radiat. 10(2), 137 (2003).

${ }^{19}$ A. C. Thompson, x-ray Data Booklet, $2^{\text {nd }}$ edition, edited by A.C. Thompson and D. Vaughan, p 4-35, LBNL/PUB-490 Rev.2, Berkeley (2001).

${ }^{20}$ K. N. Tu, C. C. Yeh, C. Y. Liu, and C. Chen, Appl. Phys. Lett. 76, 988 (2000).

${ }^{21}$ K. N. Tu, J. Appl. Phys., 94 (9), 5451 - 5473 (2003).

${ }^{22}$ J.F. Nye, in Physical Properties of Crystals: Their Representation by Tensor and Matrices, (Oxford University Press, 1957), p 25.

${ }^{23}$ P. Gilbert, R. Metzler, D. Zhou, A. Scholl, A. Doran, A. Young, M. Kunz, N. Tamura, and S. Coppersmith, J. Am. Chem. Soc., accepted for publication.

${ }^{24}$ J. M. Soler, M. Boi, J. L. Mogollon, J. Cama, C. Ayora, P. S. Nico, N. Tamura, N., and M. Kunz, Appl. Geochem. 23, 3579 (2008).

${ }^{25}$ B. C. Larson, W. Yang, G. E. Ice, J. D. Budai, and J. Z. Tischler, Nature 415, 887 (2002).

${ }^{26}$ H.A. Bale, J.C. Hanan, and N. Tamura, Powder Diffr. 21(2), 184 (2006).

27 J. R. Helliwell, J. Habash, D. W. J. Cruickshank, M. M. Harding, T. J. Greenhough, J. W. Campbell, I. J. Clifton, M. Elder, P. A. Machin, M. Z. Papiz, and S. Zurek, J. Appl. Crystallogr. 22, 483 (1989).

${ }^{28}$ Z. Ren and K. Moffat, J. Appl. Crystallogr. 28, 461 (1995). 


\section{Figure Captions:}

Figure 1: Schematic layout of the ALS beamline 12.3.2 with a superbend dipole magnet source. The beamline acceptance is $0.2 \mathrm{mrad}$ (horizontal and vertical). The toroidal M2 mirror focuses the source onto a virtual object. This intermediate image is demagnified by a ratio of $8: 1$ and 16:1 in horizontal and vertical, respectively, by a pair of Kirkpatrick-Baez mirrors. A four-bounce Si(111) monochromator can optionally be brought into the beam path for monochromatic micro-diffraction on nano-sized grains

Figure 2: Results of an online Hartmann test on the horizontal Kirkpatrick-Baez mirror. The variously coloured lines refer to different bend settings. The red line corresponds to the optimized setting and is depicted with the vertical scale in $\mu$ rad slope error in the inset.

Figure 3: Schematic of monochromator with basic measures, elevation and cross section. The channel cut $\mathrm{Si}$ crystal has an additional groove cut into the lower crystal to allow for passing the direct white beam when the crystals are set to $0^{\circ}$.

Figure 4: Schematic sketch of the sample stage. Two stacked xyz-stages allow for centring the sample onto the rotation axes $(\chi, \phi)$ and the rotation axes onto the focus spot.

Figure 5: Horizontal vibrational spectrum as measured from the intensity variation induced by a knife edge placed on the sample stage in the middle of the beam. Note the prominent modes at 40 and $45 \mathrm{~Hz}$ mainly cause by horizontal vibrations of the sample stage.

Figure 6: Measured spot shape on beamline 12.3.2. The spot size is measured by scanning a $0.15 \mathrm{~mm}$ thick Wwire through the beam and recording the absorption. The derivative of this measurement gives the spot shape. The FWHM of the horizontal and vertical focus spots are $0.63 \mu \mathrm{m}$ and $0.50 \mu \mathrm{m}$, respectively.

Figure 7: a) Calculated and observed flux curve for the monochromatic beam. Both curves have been determined with $0.15 \mathrm{~mm}(\mathrm{~h}) \times 0.1 \mathrm{~mm}(\mathrm{v})$ source slits and $1 \times 1 \mathrm{~mm}$ aperture slits.

b) Calculated flux curve for white beam configuration assuming a $8 \mu \mathrm{m}(\mathrm{h}) \mathrm{x} 16 \mu \mathrm{m}$ (v) source size, i.e. $1 \mu \mathrm{m} \times 1$ $\mu \mathrm{m}$ spot size. A $0.1 \%$ bandwidth window is assumed for the calculations. 
Figure 8: (a) Out-of-plane orientation map of the solder joint in which electrons flow from the bottom to the top and the top left corner is the current flow corner. b) Angular variation between the a-axis of the grains marked in the orientation map and the electron flow direction as a function of electromigration time. Label numbers refer to the grains numbered in a)

Figure 9: Effective resistivity of selected grains as a function of electro-migration time. Label-numbers refer to the grain numbers in $8 \mathrm{a})$.

Figure 10: Orientational spread of crystallographic $c$-axis of Aragonite platelets relative to their mean value. a) at calcite - nacre boundary, b) $15 \mu \mathrm{m}$ from boundary, c) $35 \mu \mathrm{m}$ from boundary.

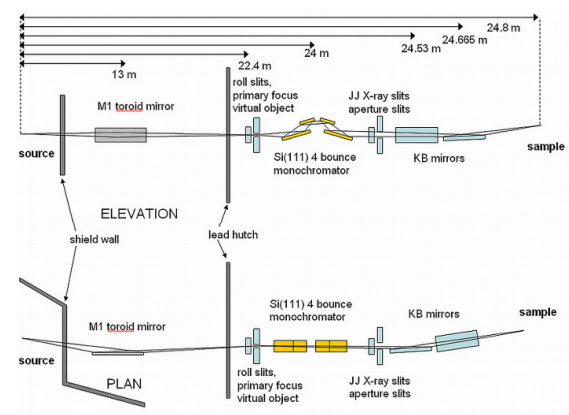

Fig. 1

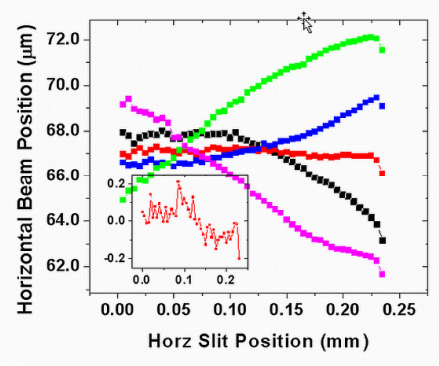

Fig. 2: 


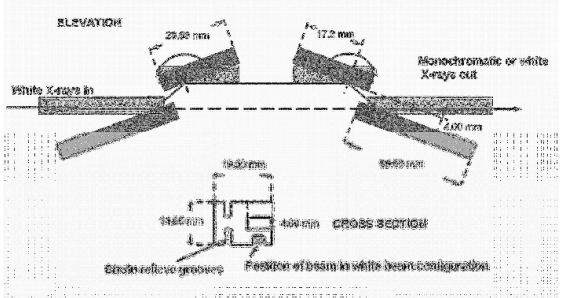

Fig. 3:

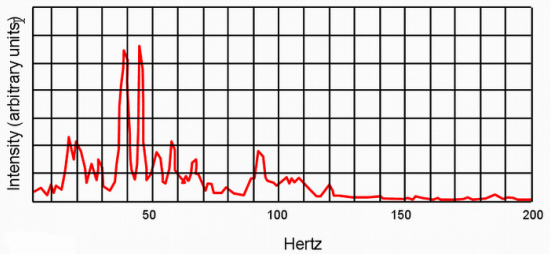

Fig. 5

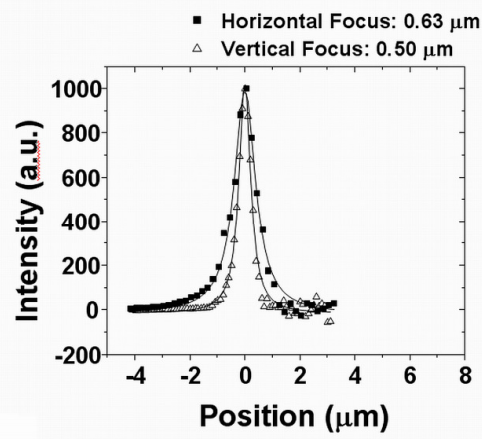

Fig. 6: 

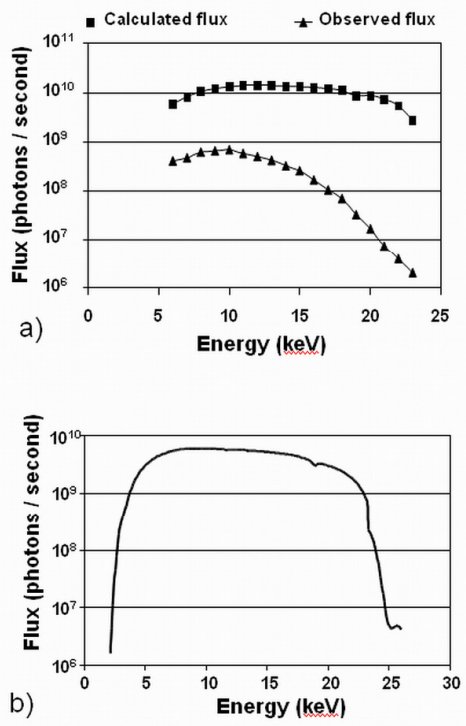

Fig. 7:
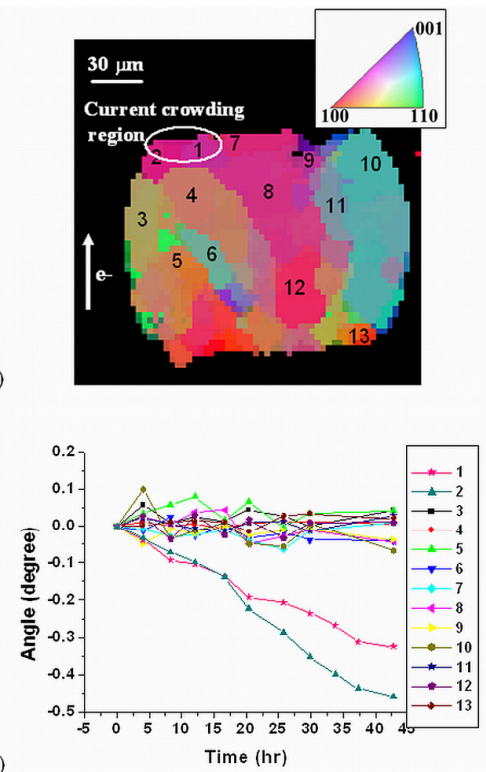

Fig. 8 


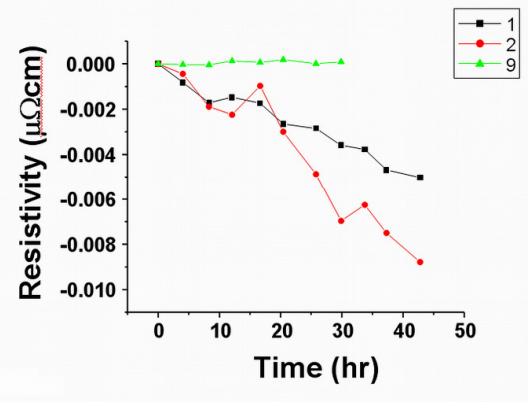

Fig. 9:

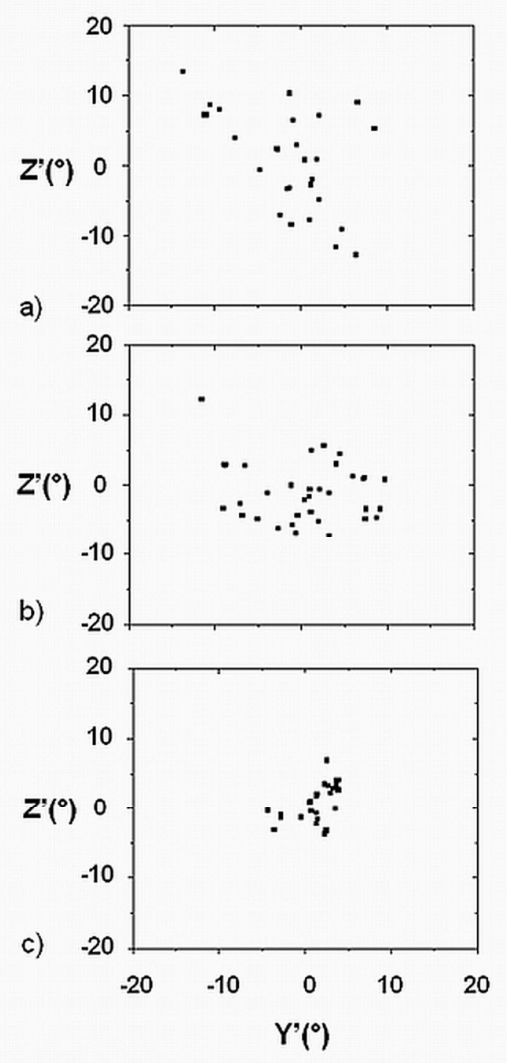

Fig. 10 